\title{
IGF-I Accelerates Ileal Epithelial Cell Migration in Culture and Newborn Mice and May Be a Mediator of Steroid-Induced Maturation
}

\author{
PHILLIP V. GORDON, JESSICA B. PAXTON, ANDREW C. HERMAN, ERICA M. CARLISLE, AND \\ NENA S. FOX \\ Department of Pediatrics, Division of Neonatology [P.V.G., J.B.P., A.C.H., E.M.C.], Department of \\ Microbiology, Tissue Culture Facility [N.S.F.], University of Virginia Health Sciences, Charlottesville, \\ Virginia 22908, U.S.A.
}

\begin{abstract}
We have previously hypothesized that IGF-I is a mediator of dexamethasone (DEX) effect in the newborn mouse ileum-a model designed to mimic the precocious mucosal maturation associated with spontaneous ileal perforations in extremely premature neonates. We have further investigated this hypothesis using in vivo and in vitro models of accelerated epithelial migration (a transient property, temporally associated with mucosal maturation). These experiments include a steroid-treatment model comparing IGF-I immunolocalization with bromodeoxyuridine (BrdU)-pulse-labeling, as a means of assessing epithelial cell migration, within the ileum of newborn mice that received either daily intraperitoneal injections of DEX $(1 \mu \mathrm{g} / \mathrm{gm})$ or vehicle. Likewise, a transgenic newborn mouse model was used to compare the effect of IGF-I overexpression upon the clearance of BrdU-pulse-labeled epithelial cells traveling up the villus during the same time period. For our in vitro model, rat ileal epithelial cells (IEC-18) were cultured to confluence in serum-free media then treated with DEX, a stable IGF-I agonist,
\end{abstract}

\section{ABSTRACT}

or nothing before being subjected to linear scarification. Serial photomicrographs of migrating cells were taken over time and the average speed was determined for each treatment condition. Our data demonstrate that IGF-I accelerates ileal epithelial cell migration in every model. However, DEX was only associated with accelerated epithelial cell migration in models where IGF-I (or a synthetic agonist) was highly abundant. In contrast, DEX by itself slowed migration speed in cell culture. These findings suggest that IGF-I may be a mediator of steroid effect during precocious maturation of the ileal mucosa. (Pediatr Res 55: 34-41, 2004)

Abbreviations
IGFBP, IGF binding protein
DEX, dexamethasone
BrdU, bromo-deoxyuridine
SFM, serum-free media

Normal mucosal maturation in rodents is triggered by a systemic cortisol surge that occurs during weaning (1). This process can be accelerated in suckling animals by exogenous administration of glucocorticoid in the weeks preceding weaning (2). However, administration of glucocorticoid in the first days of life results in a precocious maturation (characterized in the newborn mouse by mucosal growth concomitant with bowel wall thinning) (3). Further comparison between the precocious and the accelerated models of maturation in mouse pups reveals attenuated expression of maturation-related digestive enzymes in the younger animals (4). In addition to these

Received April 11, 2002; accepted May 20, 2003

Correspondence: Phillip V. Gordon, M.D., Ph.D., P.O. Box 800386, UVA Health System, Department of Pediatrics, Charlottesville, VA 22908, U.S.A., e-mail: pvg4n@virginia.edu

Supported by National Institutes of Health grants CHRCDA HD01421 (P.V.G.) and 1KO8DK/HD61553-01.

DOI: 10.1203/01.PDR.0000100461.00878.75 developmental aberrancies, precocious maturation has also been linked to a neonatal disease state.

The incidence of spontaneous ileal perforation in extremely low birth weight infants is approximately $5 \%$, but the incidence is at least twice that when DEX is administered in the first days of life (5). Like the animal models, pathology from perforated ileal specimens demonstrates hypertrophied mucosa concomitant with a thinned bowel wall (6). Immunolocalization surveys of relevant growth factors during precocious maturation in the newborn mouse ileum have implicated two probable mediators of DEX effect $(3,7,8)$. Whereas most growth factors are diminished by DEX treatment, IGF-I and transforming growth factor $\alpha$ are each redistributed from the bowel wall to the mucosa, paralleling the distribution and timing of the aberrant growth pattern.

To test the hypothesis that IGF-I is a mediator of DEX effect upon ileal epithelial cells, we sought an easily quantifiable and robust experimental outcome associated with precocious mat- 
uration [as opposed to the digestive enzymatic markers that show attenuated up-regulation in vivo and dependency upon soluble fibroblast-derived factors in culture $(4,9,10)]$. Acceleration of intestinal epithelial cell migration up the villus is an early and transient maturational phenomenon that occurs even when the near-term fetus is exposed to glucocorticoids through maternal administration (11). This acceleration of migration allows for more rapid dispersal of newly differentiated epithelial cells across the villus surface, thereby speeding functional transformation of the mucosa. To investigate the role of IGF-I in this process, we assessed ileal epithelial cell migration within in vivo and in vitro models of variable IGF-I abundance.

\section{MATERIALS AND METHODS}

Cell culture. Rat ileal epithelial cells (IEC-18, American Type Culture Collection, Manassas, VA, U.S.A.) from aliquots of passage number 6 (from original stock) were grown to confluence in SFM within six-well plates for monoculture assays. For co-culture assays, rat fibroblasts (R-12, American Tissue Culture Collection) were grown to confluence in SFM in six-well plates, seeded with IEC-18 cells, and incubated until confluent with epithelia. There was no exposure to serum or exogenous trophic factors at anytime during the two culture protocols (although IEC-18 cells were cultured with insulin during expansion, as per the supplier's protocol, then aliquoted in SFM). The time period for complete epithelial cell confluence was $48 \mathrm{~h}$ in both culture protocols.

Cultured ileal epithelial cell migration assays. Before cell culture, grids were inked upon the bottoms of the six-well plates to facilitate microscope orientation during the assays. Confluent cells were treated for $72 \mathrm{~h}$ (either with a dose of $10^{-6} \mathrm{M}$ DEX, $10^{-7} \mathrm{M}$ DEX, $10^{-7} \mathrm{M}$ long $\mathrm{R}^{3}$ IGF-I, $5 \times 10^{-7}$ $M$ long $\mathrm{R}^{3}$ IGF-I, a combination of $10^{-7} \mathrm{M}$ DEX and $5 \times 10^{-7}$ $\mathrm{M}$ long $\mathrm{R}^{3}$ IGF-I, or SFM alone (all reagents were obtained from Sigma Chemical, St. Louis, MO, U.S.A.) and then subjected to linear scarification along the midline of the grid. Scarification consisted of a single scrape of the plate with a sterile disposable pipette. The soft plastic of the pipette serves to denude the epithelia without scarring the underlying plastic and results in a uniform crawling surface surrounded on two sides by a linear border of cells. This technique is in contrast to razor blade-based protocols of wound healing, where gashes in the plastic result in a nonuniform crawling surface.

Long $\mathrm{R}^{3}$ IGF-I, rather than native IGF-I, was used because long $\mathrm{R}^{3}$ IGF-I has a much longer half-life and a significantly lower affinity for all IGFBP. These characteristics optimized receptor stimulation independently of the composition of IGFBP. It also avoided daily media exchanges, which are often necessary to demonstrate IGF-I effect in cell culture, preventing the confounder of media repletion.

Serial digital photomicrographs of individual cells were taken from three different culture dishes treated in parallel using a PixCell II laser dissection microscope (Arcturus Engineering, Mountain View, CA, U.S.A.) at 0, 6, 12, 24, 30, and $36 \mathrm{~h}$ (a time period that maximizes the assay duration but minimizes the incidence of recurrent confluence). The cells were returned to the incubator in between time points and individual assays for each cell required 2-3 min to complete. Tracking of the individual cells was accomplished by matching both the grid alignment and the residual detritus pattern within the grid across time points. This was done in real time by matching the current site to the previous digital image. A minimum of nine individual cells, three from each culture dish, were used to determine the average migration speed for a given treatment dose.

Digital images of each time point for an individual cell were contrast enhanced in Adobe Photoshop (Adobe Systems Inc., San Jose, CA, U.S.A.), laid out in sequence within Adobe Illustrator (Adobe Systems Inc.), printed along with a digital image of a micrometer (taken from the same objective), and the migration distance into the wound from time zero was measured for each time point.

In preliminary experiments, we observed that the average migration rate of IEC-18 cells varied from 7.5 to $15 \mu \mathrm{m} / \mathrm{h}$ between different batches of IEC-18 cells in monoculture, despite the fact that they were all from the same passage. Because of this variation, experiments comparing treatment conditions were performed in parallel using cells grown from the same frozen aliquot. To verify the external validity of the assays, at least three confirmatory repetitions were performed for each experiment.

Statistics. For migration assay data, the average distance from time zero was calculated for each cell at each time point and then plotted over time with SD bars. Comparisons between different treatment dosages and conditions were made using repeated measures ANOVA. Significance was defined as $p \leq$ 0.05 .

Tissue collection. The animal protocols in this study were reviewed and approved by the Institutional Animal Care and Use Committee at the University of Virginia Health Sciences and are comparable to those previously published (3). Briefly, newborn black C57 littermate mice received daily intraperitoneal injections of DEX $(1 \mu \mathrm{g} / \mathrm{gm})$ or buffered saline vehicle for either 2 or $3 \mathrm{~d}$, then were killed at $24 \mathrm{~h}$ after their last scheduled dose (thus providing two temporal points that bracket the clinical window of maximal effect with daily DEX treatment and matched controls). The chosen intraperitoneal dose is the same as the initial i.v. dose used in a prospective, randomized, multicenter clinical study where daily early postnatal DEX resulted in a dramatic increase in spontaneous intestinal perforations within DEX-treated infants (12). Intraperitoneal administration was used because reliable i.v. administration in newborn mice is not feasible.

The ileum from each animal was harvested, fixed in $10 \%$ buffered formalin, embedded in paraffin, and $4 \mu \mathrm{m}$ thick sections were cut by microtome. In some experiments, fixed ileum were sucrose protected by placing them atop a column layered with $30 \%$ sucrose on the bottom, $20 \%$ sucrose in the middle, and $10 \%$ sucrose at the top and allowing them to equilibrate at $4{ }^{\circ} \mathrm{C}$ overnight before being embedded into OCT (optimal cutting temperature compound), cryosectioned into 5 $\mu \mathrm{m}$ sections, and stored at $-80^{\circ} \mathrm{C}$.

Immunohistochemistry/immunocytochemistry. To allow direct comparisons, control and DEX-treated sections were paired together on a single slide and processed in parallel as 
previously described (3). Paraffin sections were deparaffinized in xylene and hydrated in descending ethanol concentrations before being used for immunochemistry. Cryofrozen sections were allowed to equilibrate to room temperature before being used for immunochemistry. IEC-18 cells were cultured to confluence on a coverslip in SFM, subjected to linear scarification, recultured for an additional $6 \mathrm{~h}$, and then fixed in $10 \%$ buffered formalin overnight at $4^{\circ} \mathrm{C}$ before being used for immunochemistry. Endogenous peroxide activity was quenched in $3 \% \mathrm{H}_{2} \mathrm{O}_{2}$ in $60 \%$ methanol for $15 \mathrm{~min}$ and then the slides were washed three times in distilled water.

For each condition, blocking solution $[1 \% \mathrm{wt} / \mathrm{vol} \mathrm{BSA}$ in TBS (0.5 M Tris-HCL, $0.138 \mathrm{M} \mathrm{NaCl}, 0.0027 \mathrm{M} \mathrm{KCl}, \mathrm{pH} 8.0)$ ] was applied for $1 \mathrm{~h}$ at $25^{\circ} \mathrm{C}$, followed by incubation with primary antibody for $1 \mathrm{~h}$ at $4^{\circ} \mathrm{C}$ in a humidified chamber [either rabbit anti-human IGF-I [a gift from Dr. Louis Underwood, University of North Carolina, Chapel Hill, NC, U.S.A. (13)]; mouse anti-proliferating nuclear cell antigen from Santa Cruz Biochemicals (Santa Cruz, CA, U.S.A.); or goat anti-BrdU Santa Cruz Biochemicals)]. The primary antibody solution was aspirated and the slides were washed three times in TBS before an appropriate biotinylated secondary antibody (Jackson Immunoresearch, West Grove, PA, U.S.A.) was applied for $1 \mathrm{~h}$ at $25^{\circ} \mathrm{C}$. The slides were then washed three times with TBS before ABC-horse radish peroxidase [prepared per manufacturer's instructions (Vector Laboratories, Burlingame, CA, U.S.A.)] was applied for $1 \mathrm{~h}$ and then washed as above. Peroxidase substrate solution $[0.007 \mathrm{mM}$ biotinyl tyramine, $0.003 \% \mathrm{H}_{2} \mathrm{O}_{2}$, in buffer $(150 \mathrm{mM} \mathrm{NaCl}, 100 \mathrm{mM}$ Tris-HCL, $\mathrm{pH}$ 7.5)] was applied to each section for $5 \mathrm{~min}$ and then terminated by immersion in distilled water. The sections were counterstained with hematoxylin, dehydrated, and cover slipped. Digital microscopy was performed on a PixCell II laser dissection microscope (Arcturus Engineering).

To establish an immunohistochemical marker of cell migration that could be used in each of our models, villin localization, using goat anti-villin antibody (Santa Cruz Biochemicals) and biotinylated donkey anti-goat secondary antibody (Jackson Laboratories), was performed in treated newborn mouse ileal sections and in migrating cultured ileal epithelial cells (recapitulating experiments in other gut models that have been previously published) (14-16).

Assessing ileal enterocyte migration up the villi using BrdU clearance from the proximal villus in human-IGF-I overexpressing newborn mice. Three pairs of agouti, hemizygous, metallothionine-driven, human-IGF-I overexpressing mice [originally created by Dr. Joe D'Ercole and bred to an agouti background by Dr. Kay Lund at the University of North Carolina at Chapel Hill (13)] were bred to provide paired littermates with homozygous overexpression, hemizygous overexpression, and wild-type genotypes. The litters were maintained with the hemizygous mothers (thereby standardizing the amount of IGF in the breast milk the newborn mice received within and across litters). Mice were selected by weight phenotype, similar to previously published methods, with wild-type phenotypes defined as weights in the lowest $20 \%$ of a each litter, hemizygous overexpressors as the median $20 \%$ of each litter, and homozygous overexpressors in the highest $20 \%$ of each litter at the time of euthanasia (17). These mice were weighed daily, injected with BrdU on the first day of life in the manner described above, and euthanized $48 \mathrm{~h}$ later. This protocol was designed to parallel the developmental window during which DEX-induced changes are the most striking in the newborn mouse ileum, thereby providing temporal relevance. After euthanasia, the ileum was harvested and processed for BrdU immunohistochemistry as described above, with at least one of each littermate phenotype processed at the same time on a single slide for the purpose of comparison. Photomicrographs of BrdU clearance from the proximal villus at $48 \mathrm{~h}$ after labeling were used to illustrate differences in enterocyte migration speeds between the littermates.

\section{RESULTS}

DEX increases IGF-I immunolocalization within epithelial cells. Immunolocalization of IGF-I in the DEX-treated newborn mouse ileum reveals a progressive redistribution of IGF-I from the lamina propria and basolateral spaces into ileal epithelial cells (Fig. 1). Although the absolute dosage differs between the 48- and 72-h animals, this paradigm illustrates the progression of precocious maturation after the onset of daily DEX dosing. The changes are most obvious at $48 \mathrm{~h}$ (Fig. 1A) but peak at $72 \mathrm{~h}$ in DEX-treated animals (Fig. $1 B$ ), whereas the control ileum shows a stable localization pattern within the lamina propria across the same period (a 48-h example is shown in Fig. 1C). These findings are consistent with DEXinduced internalization of IGF-I by ileal epithelial cells. Background controls were performed in parallel, with the primary antibody omitted, and minimal nonspecific staining was seen (Fig. 1D).

DEX accelerates epithelial migration in the newborn mouse ileum. BrdU labeling of ileal epithelial cells demonstrates a clustering of newly proliferated cells in DEX-treated animals when compared with controls (Fig. 2, $C$ and $D$ ). In contrast, proliferating cell nuclear antigen (PCNA) labeling shows decreased proliferation of epithelial cells per circumferential length in DEX-treated animals versus controls (Fig. 2, A and $B$ ). These findings are consistent with an accelerated upward migration of epithelial cells despite a decrease in proliferation in DEX-treated animals.

Cell migration redistributes villin to the leading edge of epithelial cells. Immunolocalization of villin in control ileum shows characteristic brush border staining, consistent with its usual localization within the microvilli of adult intestinal epithelial cells (Fig. $3 A$ ). In contrast, DEX-treatment results in a redistribution of villin to the leading edge of the epithelial cells (cell orientation is based upon the presumption that all intestinal epithelial cells are in a state of transit up the villus) (Fig. $3 B$ ). Likewise, villin abundance is increased in crawling IEC-18 cells at the wound margin and, similar to the in vivo localization, is seen intracellularly at the leading edge of the cytosol (between the nucleus and the lamellipodia) (Fig. 3D). Taken together, our in vivo and in vitro data confirm that villin immunolocalization is a sensitive means of assessing migration in vivo. 

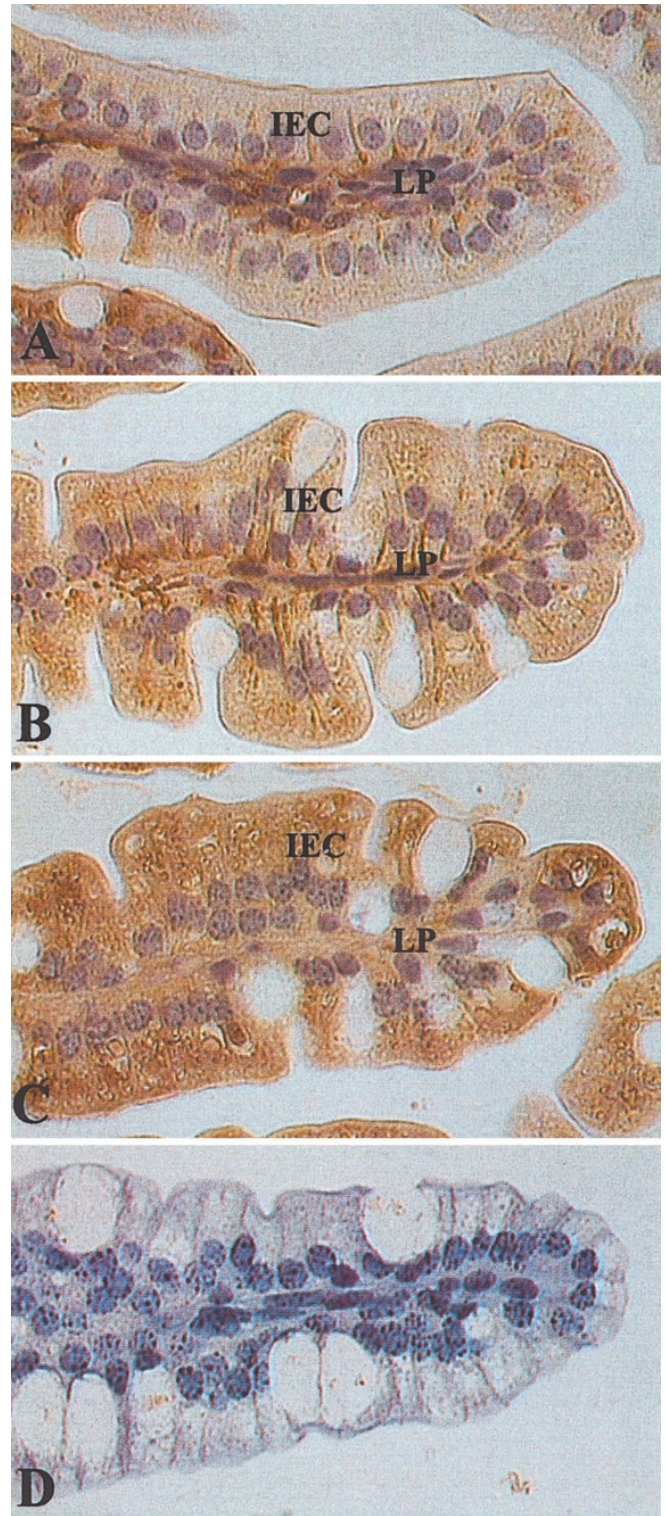

Figure 1. IGF-I immunohistochemistry in newborn mouse ileum. IGF-I localization is compared between villi from control ileum (a representative micrograph is shown at $48 \mathrm{~h}$ after onset of intraperitoneal injections of vehicle alone in $A$ ) and ileum from mice that received daily intraperitoneal injections of DEX [shown at $48(B)$ and $72 \mathrm{~h}(C)$ after onset of treatment]. These images illustrate a progressive increase in IGF-I localization within epithelial cells $(E C)$ between 48 and $72 \mathrm{~h}$ after initiation of daily DEX treatment, concomitant with a decrease of IGF-I in the lamina propria $(L P)$. In contrast, minimal change was seen in the controls during this same time period. A section of ileum after $72 \mathrm{~h}$ of DEX treatment was processed with the primary antibody omitted as a control to demonstrate that background staining was minimal $(D)$. All images were taken at $20 \times$ magnification.

\section{Individual migrating IEC-18 cells can be tracked over a} 36-h period. Our technique for measuring epithelial cell migration speed was dependent upon being able to track the same cell over a 36-h period. In part, this rigor was required because we commonly observed cell proliferation in crawling IEC-18 cells treated with long $\mathrm{R}^{3}$ IGF-I by $6 \mathrm{~h}$ after linear scarification. This confounder made assays that count the absolute number of cells migrating past a given landmark (or through a micropore) unsuitable for our purposes. In our assay, we chose the faster-

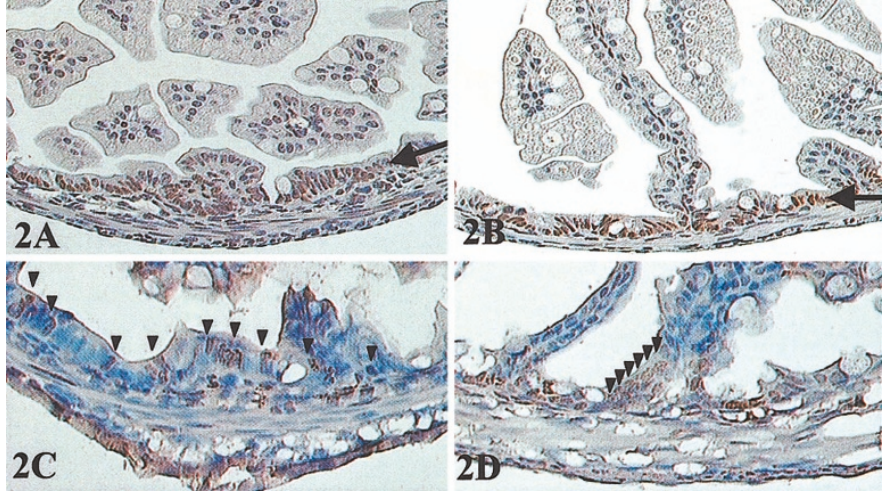

Figure 2. Immunolocalization comparisons of proliferating nuclear cell antigen (PCNA, $A$ and $B$ ) and bromo-deoxyuridine-labeled nuclei (BrdU, $C$ and $D$ ) between controls $(A$ and $C$ ) and DEX-treated newborn mice $(B$ and $D)$. A comparison between control $(A)$ and DEX-treated ileum $(B)$ after $48 \mathrm{~h}$ of treatment shows a decrease in PCNA-labeled epithelial cells within the zone of mucosal proliferation (marked by arrows) in DEX-treated ileum. In contrast, when animals treated in parallel are given a single intraperitoneal injection of $\mathrm{BrdU}$ at $8 \mathrm{~h}$ before euthanasia (control $=\mathrm{C}, \mathrm{DEX}=\mathrm{D}$ ), a separate phenomenon is evident. Controls show an even distribution of BrdU-labeling throughout the zone of proliferation whereas DEX-treated ileum shows a clustering of labeled nuclei in the villus neck, where proliferation does not occur (this is highlighted by the arrowheads in both conditions). These findings are consistent with a reduced rate of epithelial cell proliferation but an increase in the upward migration speed of daughter cells in the DEX-treated condition. PCNA-labeled images were taken at $10 \times$ magnification, whereas BrdUlabeled images were taken at $20 \times$.
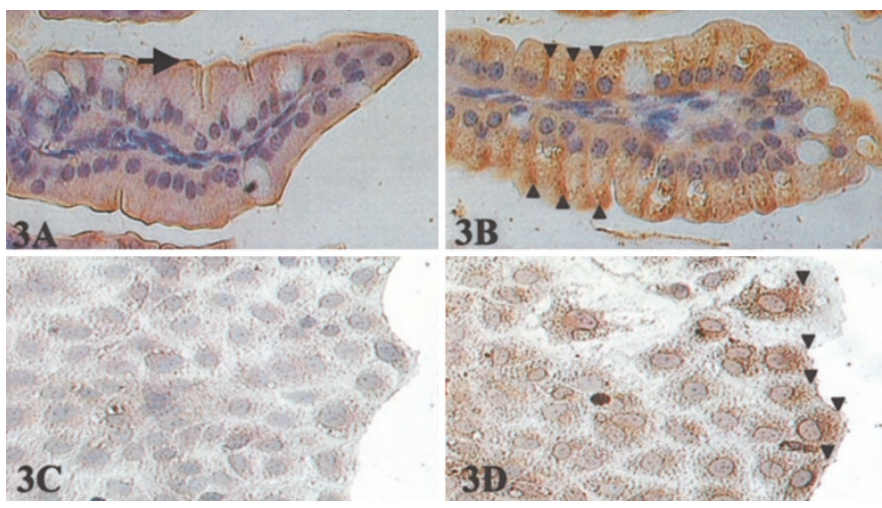

Figure 3. Villin immunolocalization in newborn mouse ileum $(A$ and $B)$ and cultured rat ileal epithelial cells $(C$ and $D)$. Villin is localized exclusively within the brush border of control ileum ( $A$, indicated by the arrow) but is redistributed to the leading edge of epithelial cells in DEX-treated ileum ( $B$, examples highlighted with arrowheads). To confirm that villin redistribution is associated with migration, rat ileal epithelial cells were cultured to confluence in SFM, subjected to linear scarification, and allowed to crawl into this wound for $6 \mathrm{~h}$ before being processed for villin immunocytochemistry. A control slide was processed without primary antibody to demonstrate that background staining is minimal with this technique $(C)$. Villin labeling in confluent epithelial cells resulted in a speckled labeling pattern throughout (consistent with discrete localization in the sparse microvilli of cultured intestinal epithelial cells), whereas cells that were migrating into the wound showed an increased abundance of villin between the nucleus and the leading edge of the cell ( $D$, examples of increased abundance are indicated by arrowheads). All images were taken at $20 \times$ magnification.

moving daughter cell in the subsequent time point and ignored the sibling cell thereafter. Our success in tracking individual cells was $94 \%$ or greater in all experiments shown in this 
article (a collage illustrating a single cell's migration over the 36-h assay is shown in Fig. 4).

DEX retards IEC-18 migration in cell culture. A 72-h treatment period with DEX resulted in a dose-dependent slowing of IEC-18 cells in monoculture (Fig. 5A). Because many aspects of DEX-induced maturation have been found to be dependent upon fibroblast-produced mediators in cell culture (18), we performed the same experiments upon IEC-18 cells that were co-cultured with R-12 fibroblasts. DEX also retarded IEC-18 migration in a dose-dependent manner within the co-culture experiments (Fig. $5 B$ ) suggesting that, unlike many other markers of maturation, the effect of DEX upon cell migration speed is not a fibroblast-dependent phenomenon.

An IGF-I agonist accelerates IEC-18 migration and rescues DEX-induced slowing. Treatment with long $\mathrm{R}^{3}$ IGF-I for $72 \mathrm{~h}$ results in a dose-dependent acceleration of migration in IEC-18 cell monoculture (Fig. 5C). To determine whether this agent could rescue DEX-induced slowing, IEC-18 cells in monoculture were treated with both $\operatorname{DEX}\left(10^{-7} \mathrm{M}\right)$ and long $\mathrm{R}^{3}$ IGF-I $\left(5 \times 10^{-7} \mathrm{M}\right)$ before performing the migration assay. This experiment resulted in a complete rescue of the DEX effect (Fig. 5D).

Overexpression of human-IGF-I is associated with accelerated enterocyte migration in the newborn mouse. Two litters of mice provided at least one full complement of phenotypes, had successful BrdU labeling, and yielded at least one ileal specimen from an animal of each transgenic condition that could be processed in parallel with its littermates. These comparisons demonstrated remarkable differences in the abundance of BrdU-labeled nuclei within the proximal portions of the ileal villi. Homozygous overexpression was associated with little to no BrdU labeling in the proximal villus, hemizygous overexpression correlated with variable numbers of labeled nuclei within the proximal villus, and wild-type animals had labeling that was evident throughout this region of the villus (representative micrographs are shown in Fig. 6). There was nearly universal staining of enterocyte nuclei in the distal villi of all animals used for comparing the transgenic conditions (data not shown), confirming successful incorporation of BrdU into the chromatin at the time of cell division. In contrast, labeling in the more proximal portions of the villus was scattered and more-often associated with goblet cells, consistent with being part of the trailing edge of the migrating BrdU-labeled enterocyte population (Fig. 6). Although these data are essentially qualitative, the clearance of BrdU-labeled nuclei from the proximal villus is proportional to the copy number of the human IGF-I transgene and likely indicates different basal rates of cell migration up the villus.

\section{DISCUSSION}

We used in vivo, transgenic, and in vitro models to investigate the hypothesis that IGF-I may mediate DEX-induced acceleration of ileal epithelial cell migration. Immunolocalization of IGF-I in the ileum of newborn mice that received daily DEX treatment showed a gradual accumulation of IGF-I in epithelial cells during the first $3 \mathrm{~d}$ of treatment. This finding is consistent with increased receptor binding and obligatory endocytosis of IGF-I. We noted that the localization of IGF-I in controls was most abundant in the lamina propria and the basolateral spaces. This predominantly extracellular distribution likely reflects the newborn serum abundance of IGF-I [which is significantly decreased by postnatal DEX exposure in human neonates (19)], prompting us to speculate that ileal epithelial cells may draw IGF-I from this systemic pool during precocious maturation and thereby contribute to a reduction in

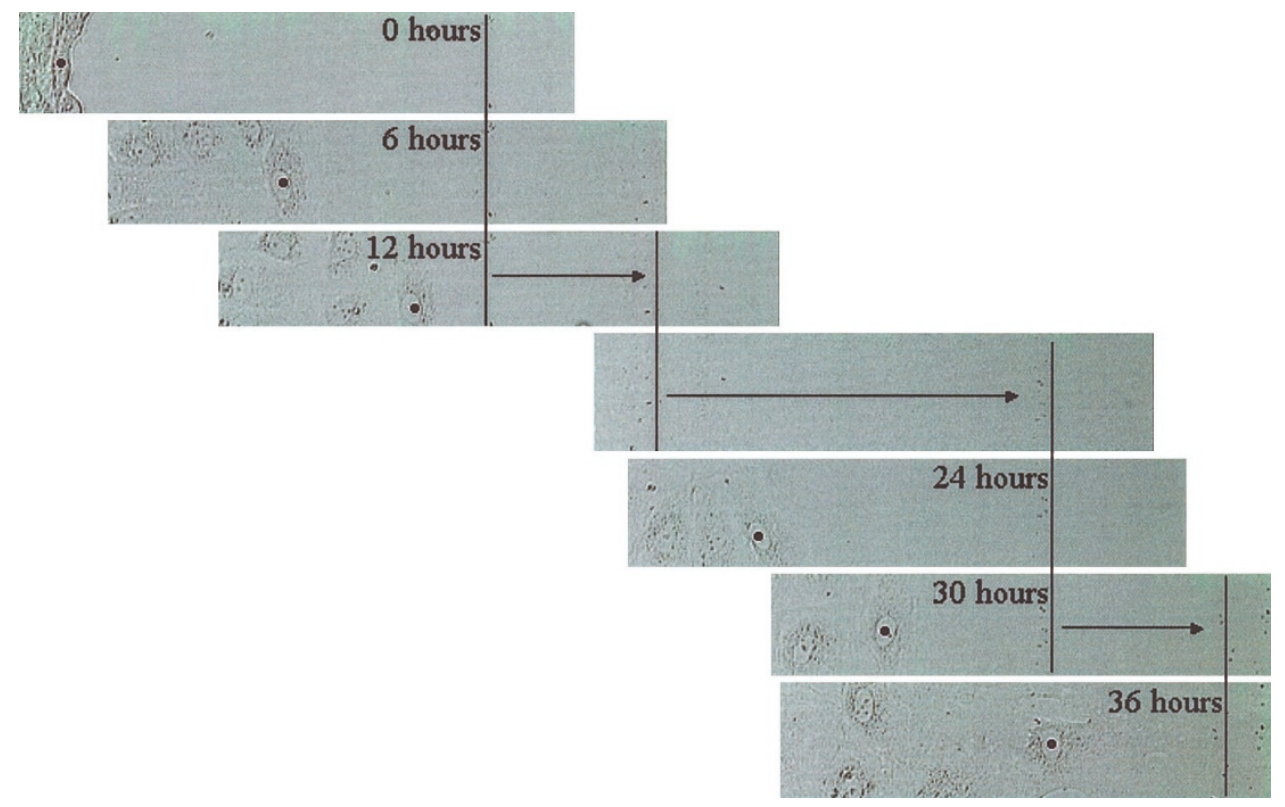

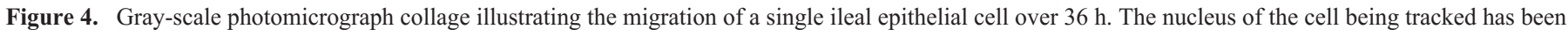

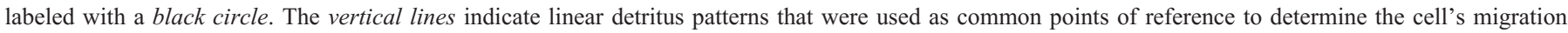

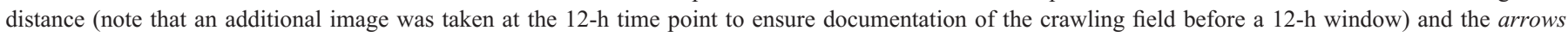
indicate time points at which a new reference point was established. All images were taken at $20 \times$ magnification. 

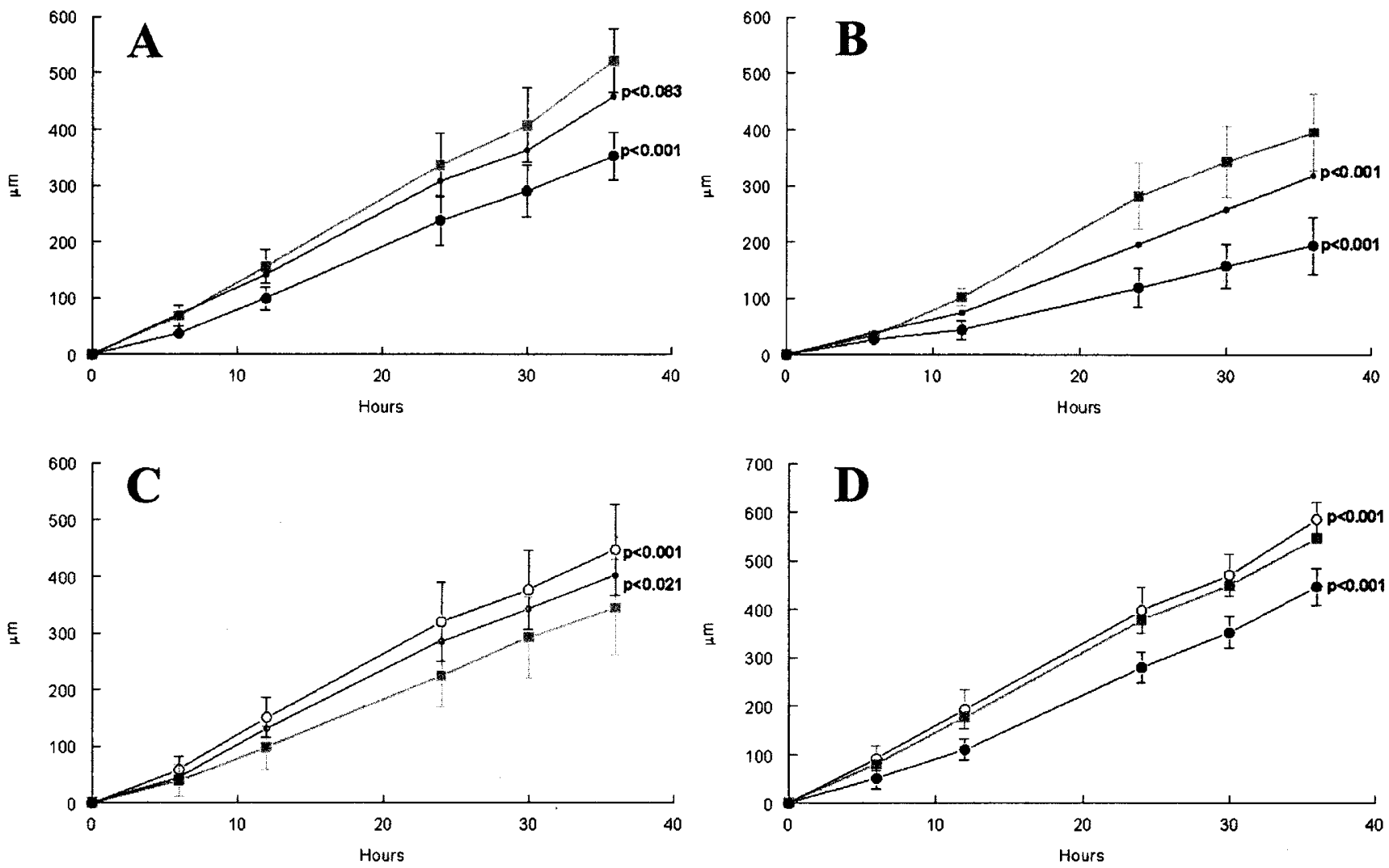

Figure 5. Graphs of ileal epithelial cell (IEC-18) migration speed in various treatment and culture conditions. $(A)$ The effect of DEX (large closed circles $=$ $10^{-6} \mathrm{M}$, smaller closed circles $=10^{-7} \mathrm{M}$ ) upon migration speed in IEC-18 culture is compared with cells cultured in SFM alone (gray squares). (B) The effect of DEX (large closed circles $=10^{-6} \mathrm{M}$, smaller closed circles $=10^{-7} \mathrm{M}$ ) upon migration speed in IEC-18 cells co-cultured atop confluent fibroblasts is compared with the same co-culture of cells in SFM alone (gray squares). (C) The effect of long $\mathrm{R}^{3}$ IGF-I (a synthetic agonist of IGF-I; large open circles $=$ $5 \times 10^{-7} \mathrm{M}$, smaller open circles $\left.=10^{-7} \mathrm{M}\right)$ upon migration speed in IEC-18 monoculture is compared with cells cultured in SFM alone (gray squares). (D) The effect of combining DEX $\left(10^{-7} \mathrm{M}\right)$ with long $\mathrm{R}^{3}$ IGF-I $\left(5 \times 10^{-7} \mathrm{M}\right)$ upon migration speed in IEC-18 culture (combination treatment $=$ open circles) is compared with DEX alone (closed circles $=10^{-7} \mathrm{M}$ ) and with SFM controls (gray squares). Differences between treatment conditions were determined by repeated measures ANOVA with significance set at $p<0.05$. Bars represent SD.
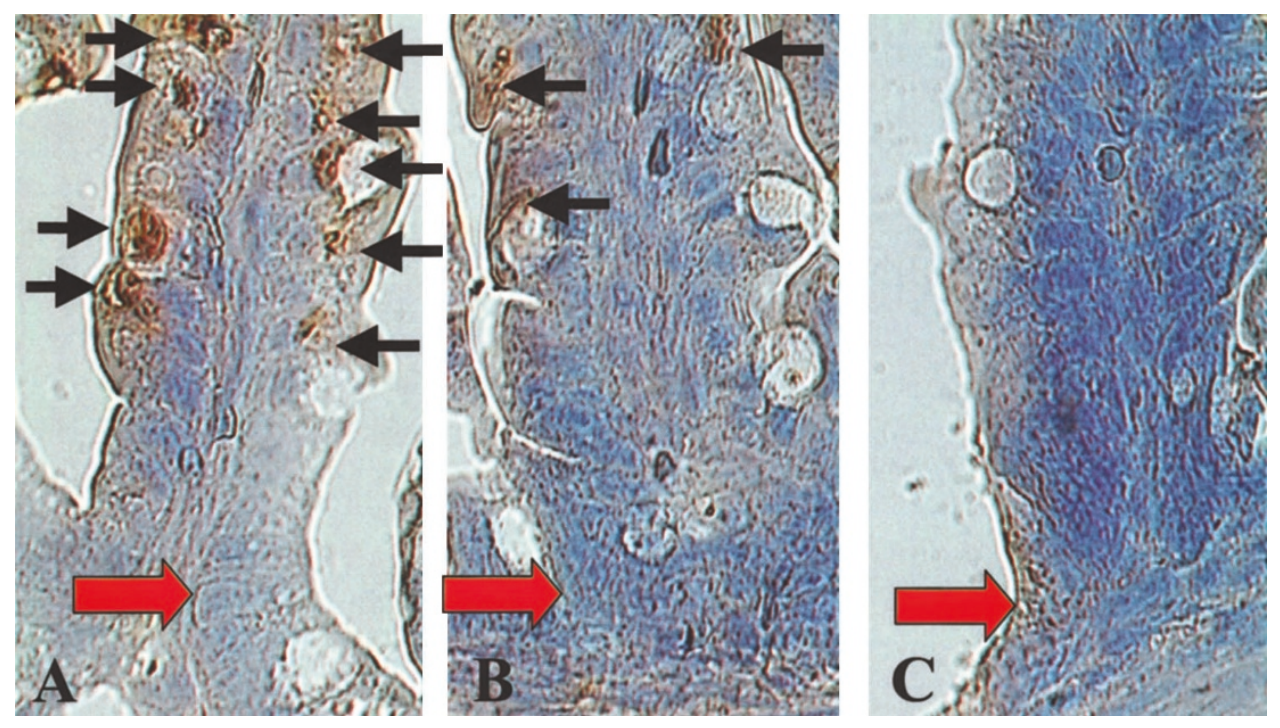

Figure 6. BrdU immunolocalization in IGF-I overexpressing newborn mice. Littermate wild-type $(A)$, hemizygous human-IGF-I overexpressing ( $B)$, and homozygous overexpressing mice $(C)$ received a single intraperitoneal injection of BrdU on the first day of life. The mice were euthanized $48 \mathrm{~h}$ later and their ileum processed for BrdU immunolocalization. BrdU immunostaining in the proximal villus (indicated with black arrows) shows a large number of labeled nuclei in wild-type mice $(A)$, a small number in the hemizygous IGF-I overexpressing mice $(B)$, and no labeling in homozygous overexpressing mice $(C)$. These findings are consistent with a positive association between IGF-I genotype and upward migration speed. In all examples, there were no labeled nuclei left within the zone of proliferation after $48 \mathrm{~h}$ (indicated by red arrows in the examples shown). All images were taken at $40 \times$ magnification. 
serum IGF-I abundance. Alternatively, IGF-I accumulation may occur via luminal uptake of IGF-I from maternal breast milk. IGF-I is highly bound by IGFBP in both serum and breast milk, so either source of mucosal IGF-I accumulation could be theoretically altered by DEX treatment (which results in mucosal accumulation of IGFBP 2, 3, 4, and 5 in the newborn mouse ileum) (20).

Corticosteroids have been shown to accelerate intestinal epithelial cell migration up the villus in late fetal development $(2,11)$. In this article, we demonstrate that DEX treatment in the newborn mouse is also associated with several markers of accelerated ileal epithelial cell migration. First, we used BrdU labeling to show clustering of newly proliferated epithelial cells in the necks of ileal villi of DEX-treated animals, despite a concomitant decrease in epithelial cell proliferation. Second, we demonstrated that villin abundance increased and its localization was redistributed from the microvilli to the leading edge of ileal epithelial cells in DEX-treated animals. A similar motility-associated phenomenon has previously been reported in epithelial cell culture and we were able to confirm these findings during ileal epithelial cell migration in cell culture as well, further supporting our supposition the ileal epithelial cells crawl faster in DEX-treated newborn mice (15). Taken together, our in vivo data are consistent with a steroid-induced acceleration of epithelial cell migration and this event is concurrent with the accumulation of IGF-I in the mucosa.

To further investigate IGF-I as a potential accelerant of ileal epithelial cell migration, we established a cell culture model of DEX effect. In contrast to in vivo models (11), DEX was found to retard IEC-18 migration in a dose-dependent fashion. In cell culture, many aspects of intestinal epithelial cell maturation are dependent upon soluble, fibroblast-produced mediators (10, 21). To determine whether DEX failed to support ileal epithelial cell migration in culture because of the absence of a fibroblast-derived factor, we performed migration assays in IEC-18-fibroblast co-cultures. DEX also retarded IEC-18 cell migration in the co-culture assays, suggesting that migration speed is not a fibroblast-dependent property but likely requires systemically available mediators of acceleration in vivo.

We examined the effect of long $\mathrm{R}^{3}$ IGF-I (a stable synthetic agonist of IGF-I) upon IEC-18 migration in monoculture and found that this agent significantly accelerated cell crawling speed. Because long $\mathrm{R}^{3}$ IGF-I accelerated epithelial cell crawling speed but DEX did not, we combined long $\mathrm{R}^{3}$ IGF-I with DEX treatment to determine which agent had the predominant effect. We found that long $\mathrm{R}^{3}$ IGF-I rescued DEX-induced slowing of IEC-18 cells, consistent with our hypothesis that IGF-I may be a mediator of DEX-effect in vivo.

As a final test of our hypothesis, littermate mice with three different IGF-I-dependent phenotypes were examined. We compared the clearance of BrdU-labeled cells from the proximal villi by immunohistochemistry at $48 \mathrm{~h}$ after a single injection between wild-type, hemizygous human-IGF-I overexpressing and homozygous human-IGF-I overexpressing littermate mice. Our qualitative assessments of BrdU clearance showed the efficiency of clearance to be proportionate to the copy number of the human IGF-I transgene. Although a more quantitative assessment would be necessary to precisely define the contribution of IGF-I as regulator of ileal epithelial cell migration speed, these findings suggest that IGF-I has an accelerant effect.

The glucocorticoid axis and the GH-IGF axis are typically thought to be opposing systemic effectors of tissue growth. However, in this article, we present three models in which increased IGF-I abundance is associated with markers or direct measures of accelerated ileal epithelial cell migration. This phenomenon has been previously associated with steroid exposure in the fetus and presumably accelerates mucosal transformation by enhancing the dispersal of ileal epithelial cells containing newly expressed digestive enzymes and signal pathways (11). Taken together, our data demonstrate an increase in mucosal IGF-I abundance during glucocorticoid-induced maturation and suggest that IGF-I may be the mediator by which steroids accelerate ileal epithelial cell migration in vivo. Additional study of this novel developmental phenomenon will expand our understanding of the pathophysiology of spontaneous ileal perforations and further characterize paracrine signaling during periods of postnatal gut maturation.

\section{REFERENCES}

1. Henning SJ, Kretchmer N 1973 Development of intestinal function in mammals. Enzyme 15:3-23

2. Nanthakumar NN, Henning SJ 1995 Distinguishing normal and glucocorticoidinduced maturation of intestine using bromodeoxyuridine. Am J Physiol 268:G139G145

3. Gordon PV, Price WA, Stiles AD 2001 Dexamethasone administration to newborn mice alters mucosal and muscular morphology in the ileum and modulates IGF-I localization. Pediatr Res 49:93-100

4. Solomon NS, Gartner H, Oesterreicher TJ, Henning SJ 2001 Development of glucocorticoid-responsiveness in mouse intestine. Pediatr Res 49:782-788

5. Gordon PV, Young ML, Marshall DD 2001 Focal small bowel perforation: an adverse effect of early postnatal dexamethasone therapy in extremely low birth weight infants. J Perinatol 21:156-160

6. Gordon P, Rutledge J, Sawin R, Thomas S, Woodrum D 1999 Early postnatal dexamethasone increases the risk of focal small bowel perforation in extremely low birth weight infants. J Perinatol 19:573-577

7. Gordon PV, Marshall DD, Stiles AD, Price WA 2001 The clinical, morphologic, and molecular changes in the ileum associated with early postnatal dexamethasone administration: from the baby's bowel to the researcher's bench. Mol Genet Metab 72:91-103

8. Gordon PV, Price WA, Stiles AD, Rutledge JC 2001 Early postnatal dexamethasone diminishes transforming growth factor alpha localization within the ileal muscularis propria of newborn mice and extremely low-birth-weight infants. Pediatr Dev Pathol 4:532-537

9. Kedinger M, Simon-Assmann PM, Lacroix B, Haffen K 1983 Role of glucocorticoids on the maturation of brush border enzymes in fetal rat gut endoderm. Experientia 39:1150-1152

10. Kedinger M, Simon-Assmann PM, Lacroix B, Marxer A, Hauri HP, Haffen K 1986 Fetal gut mesenchyme induces differentiation of cultured intestinal endodermal and crypt cells. Dev Biol 113:474-483

11. Trahair JF, Perry RA, Silver M, Robinson PM 1987 Studies on the maturation of the small intestine in the fetal sheep. II. The effects of exogenous cortisol. Q J Exp Physiol 72:71-79

12. Garland JS, Alex CP, Pauly TH, Whitehead VL, Brand J, Winston JF, Samuels DP, McAuliffe TL 1999 A three-day course of dexamethasone therapy to prevent chronic lung disease in ventilated neonates: a randomized trial. Pediatrics 104:91-99

13. Furlanetto RW, Underwood LE, Van Wyk JJ, D'Ercole AJ 1977 Estimation of somatomedin-C levels in normals and patients with pituitary disease by radioimmunoassay. J Clin Invest 60:648-657

14. West AB, Isaac CA, Carboni JM, Morrow JS, Mooseker MS, Barwick KW 1988 Localization of villin, a cytoskeletal protein specific to microvilli, in human ileum and colon and in colonic neoplasms. Gastroenterology 94:343-352

15. Nusrat A, Delp C, Madara JL 1992 Intestinal epithelial restitution. Characterization of a cell culture model and mapping of cytoskeletal elements in migrating cells. J Clin Invest 89:1501-1511

16. Andre E, Lottspeich F, Schleicher M, Noegel A 1988 Severin, gelsolin, and villin share a homologous sequence in regions presumed to contain F-actin severing domains. J Biol Chem 263:722-727

17. Pete G, Fuller CR, Oldham JM, Smith DR, D’Ercole AJ, Kahn CR, Lund PK 1999 Postnatal growth responses to insulin-like growth factor-I in insulin receptor substrate-1-deficient mice. Endocrinology 140:5478-5487 
18. Simon-Assmann PM, Kedinger M, Grenier JF, Haffen K 1982 Control of brush border enzymes by dexamethasone in the fetal rat intestine cultured in vitro. J Pediatr Gastroenterol Nutr 1:257-265

19. Bloomfield FH, Knight DB, Breier BH, Harding JE 2001 Growth restriction in dexamethasone-treated preterm infants may be mediated by reduced IGF-I and IGFBP-3 plasma concentrations. Clin Endocrinol (Oxf) 54:235-242
20. Gordon PV, Moats-Staats BM, Stiles AD, Price WA 2002 Dexamethasone changes the composition of insulin-like growth factor binding proteins in the newborn mouse ileum. J Pediatr Gastroenterol Nutr 35:532-538

21. Simon-Assmann P, Kedinger M, Grenier JF, Haffen K 1984 Organ culture of fetal rat intestine. Effects on brush border enzyme activities of the combined administration of dexamethasone and cycloheximide or actinomycin D. Enzyme 31:65-72 